\title{
The Role of Medical Word-of-Mouth on Sport Therapists: A Patient Psychological Perspectives
}

\author{
Fangyi Liu ${ }^{1}$, Ming-Yu Chou ${ }^{{ }^{*}}$,Yung-Lun Liu ${ }^{1}$, Hong-Zhou Chen ${ }^{2}$ \\ ${ }^{1}$ Department of Leisure Recreation \& Travel Management, Toko University, Chiayi County, Chinese Taipei. \\ ${ }^{2}$ Department of Animation \& Game Design, Toko University, Chiayi County, Chinese Taipei. \\ Email: *ayu@ms22.url.com.tw
}

Received June, 2013

\begin{abstract}
In 21 st century, more and more people see medical services of sport therapists as a promising industry due to global aging population and high biotechnology development. Hence, understanding medical word-of-mouth (WOM) process among patients in medical services of sport therapists could be interesting and useful. This study would find out the role of WOM on patients psychology when choosing the sport therapists, understand how patients look for sport therapists' information and which information source is important to them. As a pioneer, this study would like to improve medical WOM marketing of sport therapists. The authors designed a questionnaire based on how patients choosing sport therapists, including questions search importance of different information sources of sport therapists with medical service decision, and respondents' psychological demographics. We released 253 questionnaires of judo therapy clinics in Taiwan. More than half of the respondents see sport therapists due to others' recommendations (referral of family, friends and professionals). Women are more likely to seek medical word-of-mouth information than men; and respondents who think choosing sport therapists are important tend to seek medical word-of-mouth information. Further, patients focus on "therapist's behavior", "therapist's skills", and "therapist's ethic".
\end{abstract}

Keywords: Medical Word-of-Mouth; Sport Therapist; Patient Psychology

\section{Introduction}

Medical worth-of-mouth (WOM) of doctors or therapists reviews commonly circulate in conversations, such as chats, experience sharing, or when people ask their friends to recommend a sport therapists For example, people often make statements like "Dr. Liu is the best judo therapist in the country!" "Nobody can diagnose my problem. Do you know which therapist is an expert on this?" When people are asked about famous cardiologists, several names may enter their minds, and their impresssions usually come from hearing the discussions of others. When people cannot determine which therapist to visit, they are commonly influenced by WOM reviews, deciding to try the recommended therapists. WOM reviews are especially convincing when the receiver has heard the same WOM review regarding the same sport therapist many times, or when the spreader has substantial knowledge of sport health care, for example, people working at sport therapy clinics.

*Corresponding author.
Although the phenomena of sport therapy medical WOM reviews exist, very few sport health care-related studies have explored this issue. In the field of sport health care management, most of the studies on patients' psychology have enabled medical institutions to improve sport health care information communication by focusing on patients' psychological behaviors of seeking information regarding their symptoms and self-care after visiting doctors and therapists [1]. A significant number of studies on health care management focused on issues such as the quality of medical services, doctor-patient or therapist-patient relationships, and patients' psychological satisfaction of medical services [2]. Most of these studies focused on patients' behaviors during or after medical treatments, whereas in this study, we investigated patients' psychological behavior prior to medical treatment. After reviewing literature on WOM, we explored the influence medical WOM reviews had on patients' sport therapist selection decisions. The literature we reviewed included that on the definition of WOM, the influence of WOM on receivers' psychological decisions, and the influence of WOM on professional services. 
In addition, we limited the discussion to "the influence of medical WOM on patients' sport therapist selection" for a more focused study. We conducted surveys and interviewed patients to increase the credibility of the results. The research questions were as follows:

1) What is the significance of medical WOM on patients' sport therapist selection?

2) What are the traits of the people actively seeking medical WOM of sport therapists?

3) What are the traits of the people spreading medical WOM of sport therapist?

4) What types of WOM do patients value when they select sport therapists?

\section{Literature Review}

\subsection{Medical WOM in Health Care}

Many studies have been conducted on the WOM of service products and manufactured products, whereas few studies have been conducted on the WOM of health care services. Numerous scholars have indicated that the WOM for service products had more influence on the consumers than the WOM for manufactured products [3]. [4] also indicated that because services were intangible, consumers typically asked people with experience for information to determine whether the services met their demands and to evaluate the quality of the service. [5] emphasized the risks of service products to consumers; they argued that the purchase of service products was more risky because services were intangible, no standards could be applied, and service providers rarely offer guarantees to customers [6].

A number of scholars examine WOM in professional service sectors. For example, [7] indicated that in highrisk and highly invasive professional services, WOM was extremely significant to consumers. [8] Found that the value of WOM increased when consumers were planning to purchase service products that were complex, highly invasive, expensive, risky, and significant, such as health care services. Health care services were credence goods, which differed from search goods and experience goods. Search goods are products whose quality consumers can discern prior to the purchase; for example, consumers can try on clothing to determine whether it fits prior to purchasing. Experience goods are products whose quality consumers can judge after purchasing [9]; for example, they can determine whether the canned goods they purchase are tasty. By contrast, credence goods are products whose quality consumers have no way of judging before or after purchasing. Therefore, WOM played a significant role in the purchase of credence goods [10].

\subsection{Seeking Medical Treatment and Information}

Seeking medical treatment is a purchase psychological behavior. When patients evaluate their decisions regarding medical treatments, they use a psychological decision model similar to that of purchase behaviors, which is a series of psychological processes from demand evaluation to result evaluation after the purchase, including demand confirmation, information seeking, evaluation of alternatives, purchase psychological decision making, and afterpurchase psychological behaviors [11]. The purpose of information seeking is to obtain and compare options. This psychological process comprised internal seeking, where the consumers make judgments based on their experience and knowledge, and external search, where consumers seek external information, such as actively seeking WOM [9]. According to [12], because most patients did not understand their own psychological illnesses, they tended to seek information regarding doctors, sport therapists, clinics or hospitals from friends or relatives with a similar experience (actively seeking WOM) to save them the trouble of blind searching and to overcome their lack of sport health care information.

Several studies regarding sport health care management have been conducted on health information seeking psychological behaviors. However, these studies primarily focused on patients' psychological behaviors of seeking information on disease diagnosis and care [13]. The study by Lambert differed from this study on the point in time that was discussed: Those scholars examined how patients sought external health care information to better understand their illnesses and prepare for self-care after they had visited the sport therapists, whereas we explored how patients sought sport therapist information to determine which sport therapist to visit, that is, a pre-purchase search.

\section{Methodology and Design}

This study was conducted in cooperation with the Toko University and judo therapy clinics in Taiwan. A quantitative study was conducted at the Taiwan judo therapy clinics, where questionnaires were administered and analyzed. We selected judo therapy clinics to distribute the questionnaire and explore residents' behaviors of seeking sport medical treatment.

We distributed the questionnaires in the waiting area of the judo therapy clinics in Taiwan. Over a week, six researchers distributed questionnaires during the morning, afternoon, and evening clinic hours. The participants were randomly selected patients or the friends and families of patients. After the researchers obtained the participants' consent, the participants completed the questionnaires anonymously. The researchers assisted the elderly and visually impaired participants in completing the questionnaires.

The questionnaires were designed to study the significance of doctor WOM, who was more likely to 
actively seek medical WOM of sport therapist, whose recommendation and medical WOM were effective, and what information patients valued most when selecting sport therapists. We conducted numerous discussions, revisions, and pre-tests to obtain the final version of the questionnaire. To design the question for identifying what information patients valued the most when selecting sport therapists, we posted an open-ended question ("What do you value most when selecting sport therapists?") on social networking sites and asked internet users to nominate the top three factors they considered. We collected 125 samples and summarized the top 12 factors as follows: sport therapist's medical skills, sport therapist's attitudes, sport therapist's medical ethics, whether the sport therapists were well-known, whether the sport therapists had been involved in medical disputes, medical expenses, sport therapists' appearances, experience, education, the clinics of the sport therapist had worked at, sport therapist s' gender, and reputation.

We employed SPSS 17.0 to analyze the data, and used descriptive statistics to describe the information collected in the survey to gain an initial understanding of the influence medical WOM had on sport therapists' selection.

\section{Results and Analysis}

We administered the questionnaires outside the waiting area of judo therapy clinics in Taiwan and recovered 253 effective questionnaires. We analyzed the data using SPSS 17.0 and the results were as follows:

The age of the participants was evenly distributed, with an average of 48 years; $48.2 \%$ of the participants were male, and $51.8 \%$ were female.

Of the participants, $92.5 \%$ were local judo therapy clinics' counties, and only $7.5 \%$ were from other counties. Additionally, most of the participants (34.2\%) were college graduates.

\subsection{The Significance of WOM on Patients' Sport Therapists Selection}

WOM was significant to patients' sport therapists' selection. The patients that selected the sport therapists they would visit based on medical WOM accounted for $48.02 \%$ of the participants. Among them, 32.40\% used walk-in registration, and $19.58 \%$ used online registration. These results confirmed that reported in numerous previous studies and provided data for this study. According to [4], because service products are invisible and intangible, consumers typically ask people with experience for information regarding the products prior to the purchase, to determine whether the service suits their needs and evaluate the quality of the service. [10] Also indicated that the value of WOM increased when consumers were planning to purchase service products that were complex, highly invasive, expensive, risky, and significant, such as health care services. [14] Found that medical WOM accounted for highest percentage of American patients' pre-treatment information searching. In addition, older patients were more likely to be influenced by medical WOM when making health care decisions, because they cannot search the information on the Internet.

\subsection{The Traits the Spreaders of Sport Therapist WOM}

Recommendations from families, friends, and professsionals were the most useful. More than $70 \%$ of the participants considered recommendations from families and friends crucial, and $80 \%$ of the participants considered recommendations from medical professionals crucial, which indicated that the tie strength between the receivers and the spreaders and the professionalism of the spreaders had a significant influence on the patients [4].

\subsection{The Most Valued Factor in Patients' Sport Therapists Selection}

The most valued factor in patients' sport therapist selection was sport therapist's attitudes $(82.99 \%)$, followed by sport therapist's skills (71.16\%), medical ethics (67.19\%), reputation $(35.58 \%)$, experience $(33.20 \%)$, whether the sport therapists had been involved in medical disputes $(26.01 \%)$, the therapy clinics the sport therapists were working at $(23.71 \%)$, sport therapists' education (11.86\%), whether the doctors were well-known $(9.88 \%)$, medical expenses $(9.49 \%)$, sport therapists' gender $(2.37 \%)$, and sport therapists' appearance $(0.79 \%)$. A comparison between the answers from the participants who selected the doctors based on WOM and those who randomly selected doctors during walk-in registration showed that no significant differences in the factors they valued during sport therapists selection.

\section{Discussion and Implications}

In general purchase behaviors, when consumers considered the purchase decisions significant, they usually sought relevant information and WOM prior to the purchase [15]. However, in this study, we found that although most of the patients considered sport therapists selection crucial (74\%), only $41.5 \%$ of them actively sought WOM, whereas $59.5 \%$ of them did not. We concluded that the patients in this study tended not to actively seek medical WOM because most of them had relatively easy-to-treat illnesses (they were outpatients recruited from a clinic) and the risks were relatively low [5]; for example, patients with a cold do not need to visit a specific sport therapist. Additionally, a number of patients were unaware of the severity of their illnesses. Equally possible was that the patients had passively received 
WOM regarding the sport therapists or the patients had heard about the sport therapists from another sport therapist when the patients were transferred from one clinic to another. In both cases, the patients did not actively seek medical WOM. Patients might have also failed to actively seek medical WOM because of the lack of health care information or channels.

In this study, we enabled medical staff to understand why the patients selected them and where the patients acquired sport therapists information from. We also found that patients' behaviors of seeking medical treatment enabled the health care system and doctors to understand the sources of patients' health care information.

We concluded that patients with higher levels of medical professionalism were more likely to actively seek medical WOM. We suggest that the health care system provide additional health care education to the public to enhance their knowledge of health care. This health care system should also create more platforms for the public to exchange sport therapists' information (e.g., health care websites and forums) to improve the information flow of health care.

Most of the participants obtained information about the doctors through walk-in registration or the official Web site of the hospital, indicating that the patients had substantial trust in medical institutions in Taiwan. During the interviews, numerous participants expressed their faith in the sport therapists at clinics. Therefore, medical institutions should make it their responsibility to provide more reliable health care information to the public. We also found that the families of the patients were more active than the patients themselves in seeking medical WOM. Therefore, the sport therapists system should target their health care publicity at the families of patients. For example, many public service announcements should be designed to communicate with the families of patients.

\section{Acknowledgements}

We would like to thank the anonymous referees for their valuable comments and suggestions. The first author Fangyi Liu can be contacted at fion@ccms.nkfust.edu.tw, and the corresponding author Ming-Yu Chou can be contacted at ayu@ms22.url.com.tw.

\section{REFERENCES}

[1] J. Weaver, D. Mays, S. S. Weaver, G. Hopkins, D. Eroglu and J. Bernhardt, "Health Information-Seeking Behaviors, Health Indicators, and Health Risks," American Journal of Public Health, Vol. 100, No. 8, 2010, pp. 1520-1525. doi:10.2105/AJPH.2009.180521
[2] S. Shabbir, H. R. Kaufmann and M. Shehzad, "Service quality, word of mouth and trust: Drivers to achieve patient satisfaction," Scientific Research and Essays, Vol. 5, No. 17, 2010, pp. 2457-2462.

[3] Y. Chen, F. Liu, C. H. Fang and T. M. Y. Lin, "Understanding the Effectiveness of Word-of Mouth: An Elasticity Perspective," Journal of Research in Interactive Marketing, Vol. 7, No. 1, 2013, pp. 57-77. doi:10.1108/17505931311316743

[4] J. Bristor "Enhanced Explanations of Word of Mouth Communications: The Power of Relationships," Research in Consumer Behavior, Vol. 4, 1990, pp. 51-83.

[5] C. H. Fang, T. M. Y. Lin, F. Liu and Y. H. Lin, "Product Type and Word of Mouth: A Dyadic Perspective," Journal of Research in Interactive Marketing, Vol. 5, No. 2/3, 2011, pp. 189-202. doi:10.1108/17505931111187802

[6] F. Liu, C. H. Fang, T. M. Y. Lin and S. Chung, "Word-of-Mouth Effect of Aboriginal Cultural Products Purchase Behavior in Taiwan," International Journal of Psychology, Vol. 47, 2012, pp. 178-178.

[7] F. Liu, I. L. Chien, M. Y. Lan and S. M. Chien, "Development The Theme Park Brand Equity Model of Taiwan," International Journal of Psychology, Vol. 47, 2012, pp. 174-174.

[8] Y. Chen, F. Liu, L. M. Ho and T. M. Y. Lin, "Development of Word-of-Mouth Elasticity Measures for Tourism Product Categories," Psychology, Vol. 3, No. 9, 2012, pp. 722-728. doi:10.4236/psych.2012.39109

[9] M. Y. Lan, F. Liu, C. H. Fang and T. M. Y. Lin, "Understanding Word-of-Mouth in Counterfeiting," Psychology, Vol. 3, No. 3, 2012, pp. 289-295.

doi:10.4236/psych.2012.33041

[10] J. Sweeney, G. Soutar and T. Mazzarol, "Factors Influencing Word of Mouth Effectiveness: Receiver Perspectives," European Journal of Marketing, Vol. 42, No. 3, 2008, pp. 344-364. doi:10.1108/03090560810852977

[11] J. Engel, R. Blackwell and P. Miniard, Consumer Behavior, 8th Ed., Dryden Press, New York, 1993.

[12] H. Bansal and P. Voyer, "Word-of-Mouth Processes within a Service Purchase Decision Context," Journal of Service Research, Vol. 3, No. 2, 2000, pp. 166-177. doi:10.1177/109467050032005

[13] S. Lambert and C. Loiselle, "Health information seeking behavior," Qualitative Health Research, Vol. 17, No. 8, 2007, pp. 1006-1019. doi:10.1177/1049732307305199

[14] S. A. Nasco and D. Hale, "Information Search for Home, Medical, and Financial Services by Mature Consumers," Journal of Services Marketing, Vol. 23, No. 4, 2009, pp. 226-235. doi:10.1108/08876040910965566

[15] F. Liu and M. J. Lee, "Understanding the Island Tourism Image Formation in Taiwan: via Fuzzy Preference Relations," International Journal of Psychology, Vol. 47, 2012, p. 174. 